\title{
Single-Molecule Electron Transfer Reaction in Nanomaterials
}

\author{
Dehong $\mathrm{Hu},{ }^{*}$ Chenghong Lei, ${ }^{*}$ and Eric J. Ackerman* \\ *Pacific Northwest National Laboratory, PO Box 999, Richland, WA 99352
}

Interfacial electron transfer processes play an important role in many chemical and biological processes.[1] However, interfacial electron transfer processes are usually very complex due to high dependence on its local environments.[2][3] Here we report the study of single molecule electron transfer dynamics by coupling fluorescence microscopy at a conventional electrochemical cell. The single-molecule fluorescence spectroelectrochemistry of cresyl violet in aqueous solution and on nanoparticle surface were studied. We observed that the single-molecule fluorescence intensity of cresyl violet was modulated synchronously with the cyclic voltammetric potential scanning. We attribute the fluorescence intensity change of single cresyl violet molecules to the electron transfer reaction driven by the electrochemical potential.

In this work, an electrochemical cell was designed to allow cyclic voltammetry measurement and fluorescence microscopy measurement simultaneously. We first studied the cyclic voltammetry of $36 \mu \mathrm{M}$ cresyl violet solution and fluorescence intensity on this sample cell. Cresyl violet underwent quasi-reversible single step reduction/oxidation reaction. The fluorescence intensities of the reduced and oxidized states of cresyl violet were measured by a fluorescence microscope. The fluorescence intensity and electrochemical potential were recorded synchronously. Fig. 1 shows that the fluorescence intensity level increases and decreases with respect to ramping up and down the voltage. This result suggests that the oxidized state of cresyl violet emits strong fluorescence and its reduced state yields very weak or no fluorescence.

To examine the electrochemical dynamics of single molecules, we conducted cyclic voltammetrysingle molecule fluorescence experiments of cresyl violet adsorbed on nanoclay surface. The topography of nanoclay imaged by AFM was shown in Fig. 2. Then 120pM cresyl violet solution was used to incubate the surface. The buffer solution washed away un-adsorbed cresyl violet molecules. The fluorescence of adsorbed cresyl violet molecules were imaged by a confocal microscope. (Fig. 3) The image demonstrates that there were many cresyl violet molecules immobilized on the clay-modified surface for a long period (longer than seconds). Then the laser was focused on one molecule at a time. The fluorescence intensity of single molecules during electrochemical potential modulation was recorded. (Fig. 4) The long trajectories permit detailed statistical analyses of the on-off emission intensity of single molecules. By statistical analysis of multiple molecules, we conclude that the on-off emission intensity blinking is related to the electrochemical potential. Each emission on-off event is mainly due to the redox chemical reactions. The reduction and oxidation reaction rates were derived from the on-off emission periods and were in agreement with the ensemble averaged electrochemical measurement.

References

[1] P. F. Barbara et al., J. Phys. Chem. 100 (1996) 13148

[2] M. W. Holman et al., J. Am. Chem. Soc. 125 (2003) 12649

[3] V. Biju et al., J. Am. Chem. Soc. 126 (2004) 9374

[4] This work was supported by a grant from DOE BES. DH and CL contributed equally. 


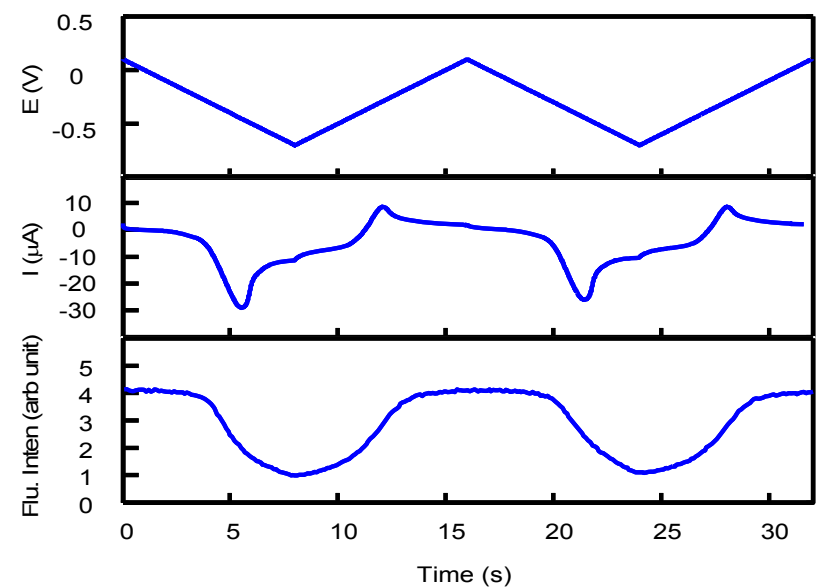

Fig. 1 Ensemble averaged fluorescence spectroelectrochemistry of $36 \mu \mathrm{M}$ cresyl violet. (Top) The voltage vs. time plot; (Middle) the current vs. time plot; (Bottom) The fluorescence intensity vs. time plot.

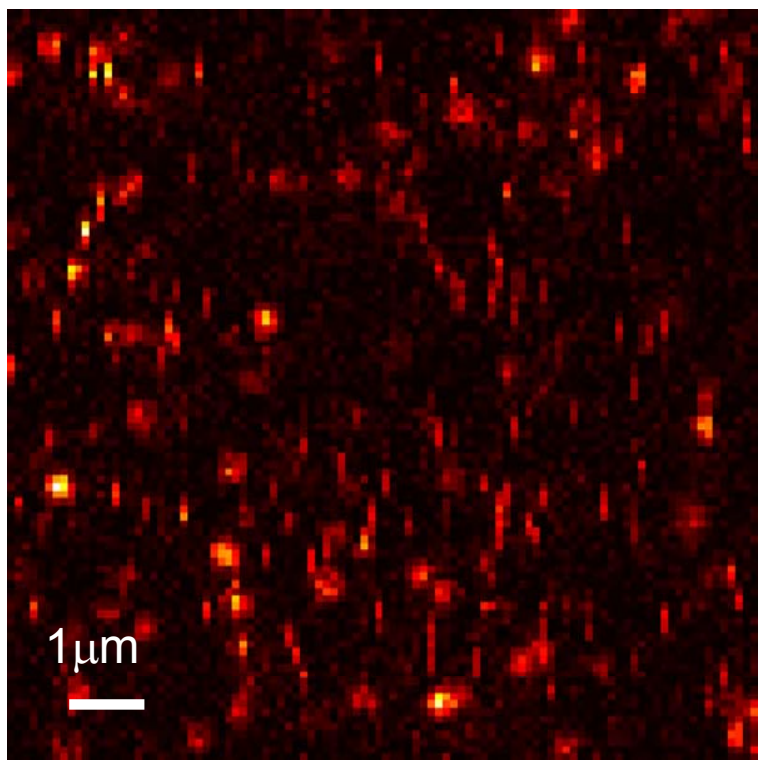

Fig. 3 The single molecule fluorescence image of cresyl violet adsorbed on clay.
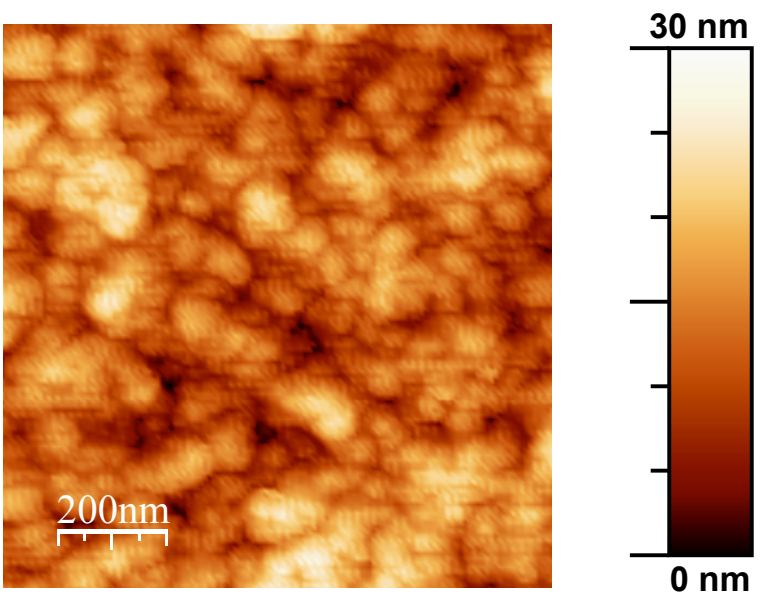

Fig. 2 AFM topography image of nanoclay on ITO glass

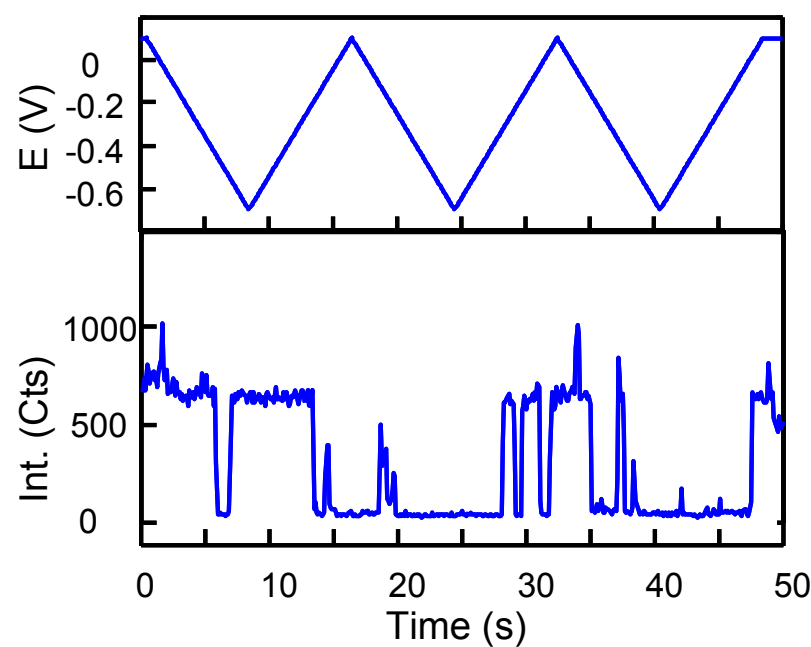

Fig. 4 Voltage E (V) vs. time plot of three cyclic voltammetry scans (top) and the plot of fluorescence intensity of a single cresyl violet molecules adsorbed on SM/ITO vs. time during the scans (bottom). 